gen gab es bei den Technologieanbietern, vor allem beim »heiligen Retter« aller Probleme, Zoom Video Communications, der im Dezember 2019 über 10 Millionen Nutzer pro Tag verfügte. Im ersten Quartal 2020 stieg die Nutzerzahl auf 300 Millionen pro Tag. Das teilweise Festsitzen im eigenen Zuhause und das Vorherrschen der virtuellen anstelle der persönlichen Kommunikation verstärkt die Einschränkungen bei der organisierten Bürgerbeteiligung, die in diesem Buch Gegenstand der Analyse sind.

\title{
Das kulturelle Gesicht der Entpolitisierung
}

Der Titel dieses Langessays suggeriert weder, dass die Staatsbürgerschaft per se von technologischen Mutationen verschlungen wird, noch, dass wir die politischen Ursachen für die Entfremdung der Demokratie vernachlässigen sollten: Wir haben es zu tun mit weitverbreiteter Korruption in Parteien, Elitismus auf Führungsebenen, und einer Krise der klassischen Konsensbildung und politischen Repräsentation. Die Vielfalt dieser miteinander verflochtenen Ebenen wird in lateinamerikanischen Umfragen ersichtlich. Das Latinobarómetro sagt aus, dass 1995 ein Drittel der lateinamerikanischen Bevölkerung an autoritären Positionen festhielt; 2018 wuchs diese Einstellung in einigen Ländern um zwei Drittel, und durchschnittlich erreicht die Zustimmung zur Demokratie nicht einmal $50 \%$.

Auch wenn Umfragen immer mit Vorsicht betrachtet werden sollten, da sie nicht die sozialen Umstände miteinbeziehen, unter denen die Antworten geäußert werden, fällt auf, dass die Tendenz zur Ablehnung der politischen Parteien und anderer Institutionen sich in so vielen lateinamerikanischen Ländern wiederholt. Daher kombinieren wir in diesem Buch quantitative Daten mit aussagekräftigen Erfahrungen aus bestimmten Situationen, die keine lineare Interpretation zulassen. Die Entbürgerlichung meint den Verlust (oder mehrere) der bürgerlichen Teilhabe im klassischen Sinne; nicht jedoch, dass zivilgesellschaftliche Aktivitäten komplett verschwinden. Es ist zum Beispiel möglich, politische Parteien abzulehnen, aber sich gleichzeitig in feministischen Bewegungen, Nachbarschafts- und Studierendenbewegungen zu engagieren.

An dieser Stelle soll über das Siechtum der Parteienpolitik sowie die Unzulänglichkeit der technokratischen Visionen gesprochen werden. Angeblich setzten gerade technokratische Ansätze darauf, die gesellschaftliche Organisation einer neutralen algorithmischen Formel anzuvertrauen. Gehen wir einmal davon aus, dass Google, Apple, Facebook und Amazon nach der Neufor- 
Grafik 1. Zustimmung zur Demokratie. Gesamtzahlen pro Land 2018.

Folgender Anteil der Befragten stimmte in den gelisteten Ländern der Aussage "Die Demokratie ist besser als jede andere Regierungsform"

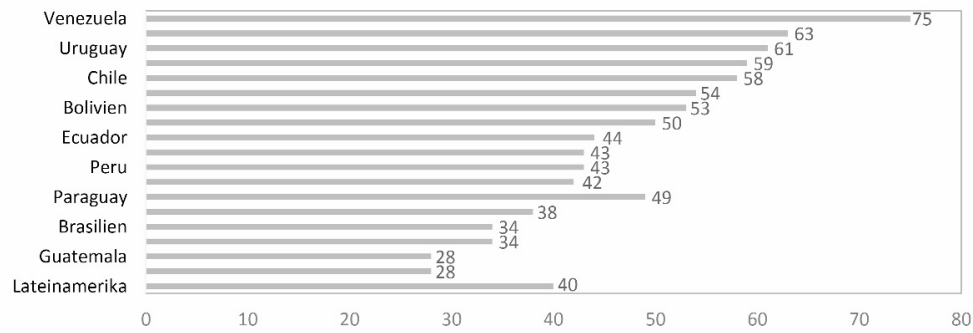

Quelle: Latinobarómetro (2018)

matierung der wirtschaftlich-politischen Macht den sozialen Sinn neu definieren: die Gewohnheiten, die Bedeutung von Arbeit, den Konsum, die Kommunikation und die Isolation der Menschen. Bei diesen Unternehmen handelt sich nicht nur um die größten Unternehmenskomplexe und wichtigsten technologischen Innovatoren unserer Zeit. Vielmehr rekonfigurieren sie die Bedeutung unseres Zusammenlebens und unserer Interaktionen. Sie zerstören das Gefühl des Zusammenlebens nach dem Verständnis der liberalen Modernisierung. Wir befinden uns jetzt jenseits der multikulturellen Fragmentierung, die die postmoderne Ära so feierte, und jenseits des Sinnpluralismus, der in den frühen Phasen der Ausbreitung des Internets und der sozialen Netzwerke konzipiert wurde.

Ein Teil dessen, was die politische Soziologie heute vor ein Rätsel stellt, wenn Phänomene wie Trump, Bolsonaro und andere Autoritarismen um sich greifen, sollte als Kultur- und Kommunikationskrise der Gesellschaft oder als neuer Weg zum Verständnis von Koexistenz, sozialer Verständigung und der Bürgerschaft untersucht werden. Der Fokus auf die Situation der Bürger ermöglicht es auch, einflussreiche, aber wenig untersuchte Phänomene zu verstehen, deren Zerstreuung es für uns Wissenschaftler unmöglich macht, sie alle gleichzeitig zu erfassen. Um die Entbürgerlichung und neu auftauchende Formen von cititzenship zu erklären, ist es notwendig, sich mit dem zu be- 
fassen, was von der bürgerlichen Teilhabe in Parteien, Gewerkschaften und religiösen und ethnischen Gruppierungen übrigbleibt. Ferner ist es wichtig $\mathrm{zu}$ sehen, was in feministischen und anderen Bewegungen, die derzeit die traditionellen Konfliktlinien, verstärkt aktiviert wird. Es ist darüber hinaus notwendig, die Rezeption literarischer und wissenschaftlicher Erzählungen in Fernsehprogrammen und Serien wie Westworld oder Black Mirror, sowie die Rezeption sozialer Netzwerke und deren diverser Nutzungsmöglichkeiten zu verfolgen. In anderen Worten geht es um globale oder regionale Akteure, die sich an Gedanken und Handlungsmöglichkeiten zur Entglobalisierung und der ökologischen Krise versuchen. Dazu stütze ich mich auf Studien, die das Wissen über diese Bereiche zwar erneuern. Angesichts der Unmöglichkeit, dieses immense Panorama vollständig zu dokumentieren und $\mathrm{zu}$ interpretieren, werde ich mich jedoch auf einzelne Szenarien und ausdrucksstarke Akteure der Prozesse in Lateinamerika und in mit der Region verbundenen Ländern stützen. Es werden Daten aus der quantitativen Forschung verwendet, wie zum Beispiel Latinobarómetro, Eurobarómetro und nationale Statistiken, sowie die neuesten Studien über die soziokulturelle Rekonstruktion der Staatsbürgerschaft in der jungen Generation und in ihrem Online- und Offlineverhalten. Aus diesen Zahlen sowie aus Ethnografien sozialer Gruppen lässt sich herausarbeiten, wie sich die Effekte der sozioökonomischen Krise und der Ausbildung digitaler Kulturen auf die subjektive Wahrnehmung sozialer Wandlungsprozesse auswirken.

Der vorliegende Text zielt damit nicht zuerst darauf, eine neue Vermessung zivilgesellschaftlichen Handelns vorzunehmen. Vielmehr geht es mir darum, kritische Dilemmata des zivilgesellschaftlichen Handelns aufzuzeigen und dessen Potential wie Beschränktheit auszuloten. Darüber hinaus möchte ich einen kritischen Blick auf das Vorgehen werfen, mit dem wir diese Dilemmata untersuchen und interpretieren, um so zivilisatorische Veränderungen zu erforschen. Ich werde insbesondere zwei Erklärungsmöglichkeiten berücksichtigen: Zum einen den biotechnologischen Determinismus und zum anderen die kritische Geschichte der Aneignung und Vermittlung von Technologie, Massenmedien und digitalen Netzwerken. Als weiteres Fundament dienen qualitative Studien, die die charakteristischen Krisen in Lateinamerika untersuchen. Für einen verständlichen und überschaubaren Leseprozess konzentriere ich mich auf die wichtigsten Knotenpunkte und verweise auf eine abschließende Bibliographie, um so im Sinne eines Essays die Freude am Lesen und Reflektieren anzuregen ohne die akademische Exaktheit zu sehr zu vernachlässigen. 